\title{
On the Status of Irreducibility Problems of Open Shop Sequencing Problems
}

\author{
Tanka Nath Dhamala \\ Central Department of Mathematics, Tribhuvan University, Kirtipur, Kathmandu \\ e-mail:dhamala@yahoo.com
}

\begin{abstract}
In an open shop scheduling problem (OSSP) one of the major tasks is to minimize the makespan over the sequences. It has practical values, for instance, in manufacturing, service industries, communication, and facility assignments. The concept of irreducibility has proven one of the most important and fertile research topics as the set of all irreducible sequences attains optimality independent of processing times. We present the recent complexity status of irreducibility of OS sequences. The recent works give encouraging results. The results obtained in past several years are viewed with useful hints for the developments of exact or heuristic algorithms and generalize the idea to general regular objectives.
\end{abstract}

Key words: scheduling/sequencing theory, open shop, reducibility, potential optimality, complexity

\section{Introduction}

We consider the strongly NP-hard OSSP $O \| C_{\max }$ (Gonzalez \& Sahni 1976), which has been mostly considered (see, Andresen et al. 2008). In the scheme $\alpha|\beta| \gamma, \alpha, \beta$ and $\gamma$, respectively, represent the machine environment, job characteristics and optimality criterion. In a preemptive schedule, a job can interrupt its processing and complete the remaining part in later time on the same machine. Each job $i$, with $i \in I=\{1, \ldots, n\}$ has to be processed on each machine $j$, with $j \in J=\{1, \ldots, m\}$ exactly once without preemption for the positive time such that, at a time, each machine can process at most one job and each job can be processed on at most one machine. The problem $O 2 \| C_{\max }$ is solvable in linear time (Gonzalez \& Sahni 1976, Braesel \& Kleinau 1996).

The order in which a job is processed on machines is called machine order and the order in which a machine processes jobs is called job order. It is an OS if all processing orders are arbitrarily. An optimizer seeks to find a sequence (feasible combination of all job orders and machine orders) which minimizes the makespan. A schedule is the corresponding timetable. A sequence is optimal if it yields a schedule with minimum objective value from the feasible set. Let
$S I J=I \times J, P=\left[p_{i j}\right]_{n \times m}$ and $C=\left[c_{i j}\right]_{n \times m}$ be the sets of all operations $o_{i j}$, and matricies of processing times $\mathrm{p}_{\mathrm{ij}}$ and of completion times $c_{i j}$, respectively. The objective function is $C_{\max }=\max _{i} C_{i}=\max _{i j}\left(c_{i j}\right)$, where the completion time $C_{i}$ is the completion time of job $i$. The set of all instances of $P=\left[p_{i j}\right]$ is denoted by $P_{n m}$. Here, $C_{\max }(A)$ represents the finish time of the schedule $C=(A, P)$ for the matrix $P$ and the sequence $A$.

A schedule is called semiactive if each operation is started as early as possible with respect to the given processing orders. For a regular objective, one can restrict the investigation in the space of all semiactive schedules. Mapping the associated semiactive schedule $C$ for a given $A$ and $P$ is an easy problem. The difficulty on the complexity issues of the OSSP lies to the construction of an appropriate sequence. Therefore, the study concentrated either on the determination of polynomial solvable subproblems or on the development of an algorithm for an approximate solution.

Determination of the feasible solution space by considering the cardinality of special latin rectangles or the chromatic polynomial of the Hamming graph $K_{n} \times K_{m}$ is a difficult counting problem (Harborth 1999). 
Nepal Journal of Science and Technology 11 (2010) 205 - 214

A closed formula for this unsolved counting problem is unlikely in general. However, bounds are available in general cases (Braesel \& Kleinau 1992a, 1999b, Dhamala 2002). Enumerative results demonstrate a huge number of sequences in OSP (Braesel et al. 1999a, 1999b, Harborth 1999).

A set of sequences is potentially optimal if it contains an optimal sequence with respect to the given objective function for arbitrary data. The elements of such a solution set are potentially optimal. Obviously, the set of all sequences is potentially optimal thought not the minimal one. Existence of unique minimal potentially optimal set is unlikely, in general.

Reducibility is very applicable when the processing times are erroneous, difficult to find out in advance or simply unknown in manufacturing and service industries. For example, a car may require repairs on its engine, body and electrical circuit in a large automotive garage with specialized shop centers. These operations may be processed in any order but it is not possible to perform any two of the same job simultaneously. Similar applications of OSSP may arise in testing components of an electronic system, repairing parts of an airplane in a large aircraft garage and satellite communications (Prins 1994). The OS arises in many industrial environments like quality control centers, semiconductor manufacturing including the applications in examination scheduling and teacher class assignments (Andresen et al. 2008).

The irreducible sequences by introducing a dominance relation on the set of all sequences with a fixed format $\mathrm{n} \times \mathrm{m}$ have been studied since last 20 years (Kleinau 1993). The irreducible elements are the minimal sequences with respect to this partial order independent of the given processing times. The solution set of all these locally optimal sequences is potentially optimal of smaller cardinality. The irreducible sequences for the problem $O \| C_{\max }$ on an operation set with spanning tree structure are studied in (Braesel \& Kleinau 1996). This concept to minimize the makespan have been studied in (Dhamala 2008). A necessary and sufficient condition for the irreducibility can be tested in polynomial time on tree-like operation sets (Tautenhahn \& Willenius 2000).

The irreducibility is generalized by considering a dominance relation between a sequence and a set of sequences (Tautenhahn \& Willenius 2000, Willenius
2000). A sequence $A$ is unavoidable with respect to a sequence set if for all sequences in this set, there exist processing times with a better value on $A$. They give several necessary and sufficient conditions for a sequence to be dominated by a set. They formulate the dominance relation as a mixed integer program and compute minimal potential solutions for small sized OSP. The results on irreducibility for other regular objectives are extended in (Willenius 2000). A sequence decomposition approach is introduced in (Dhamala 2002, Dhamala 2007).

Problems with uncertain input data under uncertainty have been considered under stability analysis calculating the stability radius. This radius is the largest quantity of independent variations of the data such that an optimal schedule remains optimal. It is used for the phase of an algorithm at which a sequence of a scheduling problem has already been found and more calculations are made in order to investigate how this sequence depends on the data. There are relations between the stability and irreducibility analysis (Sotskova 2001, Harborth 1999).

Up to today, no polynomial time algorithm is known for the decision whether a sequence is irreducible in the general case. Recently, two variants of algorithms based on the specific properties of the corresponding H-comparability graph are presented and thus the problem of irreducibility is solved partially in (Andresen 2009, Dhamala 2010, Andresen \& Dhamala 2010). The first variant is polynomial whereas the second is exponential. They expose a number of open problems.

Section 2 reviews the mathematical formulation of shop graphs and their main properties. We give brief sketch of irreducibility and reducibility in Section 3 . It also considers the minimal potentially optimal solution sets and gives the main idea of recent reducing algorithms. Last section concludes the paper.

\section{Fundamental Properties The Sequence Graph}

Here, we use the block-matrices model (Braesel 1990). Special latin rectangles are used to formulate this model which are also called sequences. A latin rectangle $L R[n, m, q]=\left[l r_{i j}\right]$ is a matrix of size $n \times m$ with $l r_{i j} \in\{1, \ldots, q\}$ such that each integer of the symbol set $\{1, \ldots, q\}$ 
Tanka Nath Dhamala/On the Status of Irreducibility........

occurs at most once in each row and in each column of $L R$. If $n=m=q$, then the matrix is a latin square of order $n$ (Dhamala 2002).

The $n \times m$ matrices of all job orders and machine orders are denoted by $J O$ and $M O$, respectively. By $m o_{i j}=k$ we mean that $o_{i j}$ is the $k$-th operation of job $i$ in the machine order. For any $(M O, J O)$, we define the shop graph $G_{M O, J O}=(S I J, E)$ where $E$ represents the precedence constraints between the operations. A shop graph is a sequence graph, which is an acyclic orientation of the disjunctive graph, (nonsequence graph) if it is acyclic (cyclic). An efficient algorithm of complexity $O\left(\max \left\{m n^{2}, m^{2} n\right\}\right)$ to decide whether a given connected digraph is a shop graph (sequence graph) is given in (Dhamala 2002, Braesel et al. 2001).

For each sequence graph we can describe the sequence $(M O, J O)$ by a special latin rectangle $A=$ $\left[a_{i j}\right]$, where $a_{i j}=\operatorname{rank}\left(o_{i j}\right)$, with sequence property: for each integer $a_{i j}>1$ there exists $a_{i j}-1$ in row $i$ or in column $j$ or in both. An arc from $o_{i j}$ to $o_{\mathrm{kl}}$ exists if and only if $i=k$ or $j=l$ is satisfied and $a_{i j}<a_{k l}$ holds.

Example 2.1 The machine orders for the jobs $\mathrm{J}_{1}: \mathrm{M}_{1}$ $\rightarrow \mathrm{M}_{2} \rightarrow \mathrm{M}_{3}, \mathrm{~J}_{2}: \mathrm{M}_{1} \rightarrow \mathrm{M}_{3} \rightarrow \mathrm{M}_{2}$, and the job orders on the machines $\mathrm{M}_{1}: \mathrm{J}_{1} \rightarrow \mathrm{J}_{2}, \mathrm{M}_{2}: \mathrm{J}_{1} \rightarrow \mathrm{J}_{2}$, $\mathrm{M}_{3}: \mathrm{J}_{2} \rightarrow \mathrm{J}_{1}$, yield the following matrices

$$
\begin{gathered}
\mathrm{A}=\left(\begin{array}{lll}
1 & 2 & 4 \\
2 & 4 & 3
\end{array}\right), \\
\mathrm{MO}=\left(\begin{array}{lll}
1 & 2 & 3 \\
1 & 3 & 2
\end{array}\right), \mathrm{JO}=\left(\begin{array}{lll}
1 & 1 & 2 \\
2 & 2 & 1
\end{array}\right)
\end{gathered}
$$

By $\mathrm{A}_{-1}$ we mean the reversed sequence constructed from $\mathrm{A}$ with all arcs in the reversed direction.

Theorem 2.1 There is a one-to-one correspondence between the sets of all sequences and all sequence graphs. The mapping is in linear time on the number of operations.

For this, one calculates the rank matrix (latin rectangle) to each vertex of the sequence graph, and on the other hand, also constructs a sequence graph with labeling the integers of a latin rectangle (Braesel 1990).

\section{Counting Sequences}

It holds, $\left|S_{n m}\right|+\left|S^{*}{ }_{n m}\right|=(m !)^{n}(n !)^{m}$ for the sequence set $S_{n m}$ and the nonsequence set $S^{*}{ }_{n m}$ of $n \times m$ the shop graphs in OS. Exact formula for 2-jobs and better bounds for the exact algorithms for higher order sequences are given in (Kleinau 1993, Braesel \& Kleinau 1992). Moreover, exact numbers are calculated for $n=2 \wedge(2 \leq m \leq 8)$ and $n=3 \wedge(3 \leq m \leq 4)$ (Braesel \& Kleinau 1992), and for $n=3 \wedge(5 \leq m \leq 7)$ and $n=4 \wedge(4 \leq m \leq 5)$ in (Braesel et al. 1999a, 1999b).

An infinite set of schedules can be assigned to each sequence. We can define an equivalence relation on the set of all schedules decomposing the set into finite number of classes. Two schedules belong to the same class if and only if they base on the same sequence. The semiactive schedules under unit processing times, i.e., a finite set of all sequences, are used to find a set of distinct representatives.

One way, to deal with the cycle space $S^{*}{ }_{n m}$ is to count the fundamental cycles in the shop graph. Each subgraph of $K_{n} \times K_{m}$ induced by the operations $\left\{\boldsymbol{o}_{\mathbf{i 1 j} 1}\right.$,

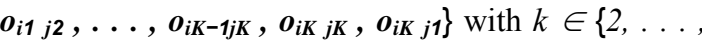
$\min \{m, n\}\}, i_{u}=i_{v}$ and $j_{u}=j_{v}$ for all $u$, v with $u=v$, is called a fundamental cycle $\left[C_{2 k}\right]$ of length $2 k$. Each $\left[C_{2 k}\right]$ alternately contains edges of $K_{m}$ and $K_{n}$. There are $2^{2 k}$ different orientations of $\left[C_{2 k}\right]$ but only two of them are fundamental dicycles.

Each nonsequence graph contains at least one such a dicycle. For fixed $k \in\{2, \ldots, \min \{m, n\}\}$, The number of dicycles which contain at least one fixed fundamental dicycle is given in (Dhamala 2002). Moreover, a general formula for the number of all sequences is developed. First question would be: how can a set of pairs $(M O, J O)$ be constructed with exactly given number of prescribed dicycles?

Theorem 2.2 Let $L S_{1}, L S_{2} \in L S[n]$. Then, a necessary and sufficient condition that the pair $\left(L S_{l}\right.$, $L S_{2}$ ) is a sequence is that they are identical.

\section{Irreducibility}

A given sequence $A$ is called reducible to another sequence $\mathrm{B}$ if $C_{\max }(B) \leq C_{\max }(A)$ for all $P \in P_{n m}$, denoted by $B \preceq A$. Clearly, $B$ is optimal if $B \preceq A$ for all 
Nepal Journal of Science and Technology 11 (2010) 205 - 214

$A$, though an optimal solution may not be unique. The equality $C_{\max }(A)=C_{\max }\left(A_{-1}\right)$ holds. A sequence $A$ is called strongly reducible to $B$, denoted by $\mathrm{B} \prec \mathrm{A}$, if $B$ $\preceq A$ but not $A \preceq B$. Two sequences $A, B$ are called similar, $A \simeq B$, if $B \preceq A$ and $A \preceq B$. A sequence is irreducible if there exists no other non-similar sequence to which it can be reduced.

The set of all $n \times m$ irreducible sequences is denoted by $S_{n m}^{I}$. The irreducible elements are the minimal sequences with respect to the partial order $\prec$ and hence are locally optimal. This relation drastically reduces the set of all sequences which must be considered. The similarity relation $\simeq$ is an equivalence relation on $S_{n m}$ decomposing this set into disjoint equivalence classes. The set of all pairwise non-similar irreducible sequences, denoted by $\mathrm{S}_{\mathrm{nm}}^{\mathrm{I}}$, is potentially optimal and $\left|\mathrm{S}_{\mathrm{nm}}^{\mathrm{I}} *\right| \leq \frac{1}{2}\left|\mathrm{~S}_{\mathrm{nm}}^{\mathrm{I}}\right|$ holds.

\section{Maximal Paths}

A path $w_{A}$ with vertex set $V\left(w_{A}\right)$ in the sequence $\mathrm{A}$ is called maximal if there does not exist another path $w_{A}^{*}$ with $V\left(w_{A}\right) \subset V\left(w_{A}^{*}\right)$. The set of all maximal paths in $A$, denoted by $W_{A}$, contains an exponential number of maximal paths. One of the paths in $W_{A}$ becomes the longest depending on processing times. The $A$ reduced to $B$ if and only if for all $w_{B} \in W_{B}$ there exists $w_{A} \in W_{A}$ such that $V\left(w_{B}\right) \subseteq V\left(w_{A}\right)$. If $B \prec A$, then there exists $w_{B} \in W_{B}$ such that $V\left(w_{B}\right) \subset$ $V\left(w_{A}\right)$ for some $w_{A} \in W_{A}$. The relation $B \prec A$ does not necessarily imply $C_{\max }(B)<C_{\max }(A)$ for arbitrary $\mathrm{p}_{\mathrm{ij}}$. The strict inequality remains true if there exists a unique maximal path in $A$. The following matrices illustrate more explanation.

$$
\begin{aligned}
& A=\left(\begin{array}{lll}
1 & 2 & 4 \\
2 & 4 & 3
\end{array}\right), B=\left(\begin{array}{lll}
4 & 1 & 3 \\
1 & 3 & 2
\end{array}\right), \\
& C=\left(\begin{array}{lll}
2 & 1 & 3 \\
1 & 3 & 2
\end{array}\right), P=\left(\begin{array}{ccc}
1 & 1 & 10 \\
1 & 1 & 1
\end{array}\right)
\end{aligned}
$$

We have $A \succ B$ (respectively, $B \succ C$ ) since $\left\{o_{11}, o_{21}\right.$, $\left.o_{22}\right\}$ (respectively, $\left\{o_{23}, o_{13}, o_{11}\right\}$ ) belong to a common path in $A$ (respectively, in $B$ ) but not in $B$ (respectively, in $C$ ), and whenever certain operations belong to a common path in the latter sequence these operations also belong to a common path in the former. Moreover, $C_{\max }(A)=C_{\max }(B)=13$ but $C_{\max }(C)=12$ for given $P=\left[p_{i j}\right]$.
An undirected graph $G=(V, E)$ is called a comparability graph if there exists an acyclic orientation $E^{t r}$ of $E$ such that the corresponding digraph $G^{t r}=\left(V, E^{t r}\right)$ is transitive closure, i.e. $(a, b)$ $\in E^{t r}$ and $(b, c) \in E^{t r} \quad$ implies that $(a, c) \in E^{t r}$ for all arcs. The comparability graph of a sequence graph $G_{A}$ is denoted by $\left[G^{t r}{ }_{A}\right]$, where $[G]$ stands for the underlying undirected graph of a digraph $G$ and $G^{t r}=\left(V, E^{t r}\right)$ denotes the transitive closure of $G$, (Dhamala 2002).

\section{Sequence Implication Classes}

An $\operatorname{arc}\left(o_{i j}, o_{k j}\right)$ in $A$ is said to directly imply $\left(o_{i j}, o_{i l}\right)$ in the same sequence if and only if $\left\{o_{k j}, o_{i l}\right\} \notin\left[\mathrm{E}_{\mathrm{A}}{ }^{\text {tr }}\right]$. Similarly, we have $\left(o_{i l}, o_{k l}\right) \gamma\left(o_{k j}, o_{k l}\right)$ in sequence $A$ if and only if $\left\{o_{i l}, o_{k j}\right\} \notin\left[\mathrm{E}_{\mathrm{A}}{ }^{\mathrm{tr}}\right]$. If $\left\{\mathrm{o}_{\mathrm{il}}, \mathrm{o}_{\mathrm{kj}}\right\} \notin\left[\mathrm{E}_{\mathrm{A}}{ }^{\mathrm{tr}}\right]$, then $\left\{o_{i l}, o_{k j}\right\} \notin\left[\mathrm{E}_{\mathrm{B}}{ }^{\text {tr }}\right]$ whenever $A$ is reduced to $B$. An arc $\left(o_{i j}, o_{u v}\right)$ in a sequence $A$ is said to imply an $\operatorname{arc}\left(o_{k l}\right.$, $\left.o_{x y}\right)$ in the same sequence, denoted by $\left(o_{i j}, o_{u v}\right) \gamma^{\text {tr }}$ $\left(o_{k l}, o_{x y}\right)$, if there exists a chain of $\operatorname{arcs} e_{1}, e_{2}, \ldots, e_{k}$ in $A$ such that $\left(o_{i j}, o_{u v}\right) \gamma e_{1} \gamma e_{2} \ldots e_{k} \gamma\left(o_{k l}, o_{x y}\right)$ holds. The relation $\gamma^{\text {tr }}$ is an equivalence relation partitioning the arc set of sequence graph into disjoint equivalence implication classes in $O\left(n^{2} m^{2}\right)$ time and space (Willenius 2000). We call these classes by sequence implication classes.

Note that a graph is a comparability graph if and only if there is no implication class containing both an arc and its reverse. A sufficient condition for irreducibility is presented in terms of implication classes (Braesel et al.1999a, Willenius 2000).

\section{Algebraic Structures}

Let $S_{t}$ be the symmetric group of order $t$ and $Z_{2}$ be the cyclic group of order two. Consider a row permutation $\pi_{r} \in S_{n}$, a column permutation $\pi_{\mathrm{c}} \in \mathrm{S}_{\mathrm{m}}$, a transposition $\Phi \in Z_{2}$, and a reversion $\Psi \in Z_{2}$ of a matrix, respectively. Two given sequences $A$ and $B$, they are called structure isomorphic, graph isomorphic or permutation isomorphic, denoted by $\cong_{\mathrm{s}}, \cong_{\mathrm{g}}$ or $\cong_{\mathrm{p}}$ if there exists a mapping such that $\left(\pi_{r}, \pi_{c}\right.$, $\Phi, \Psi) A=B,\left(\pi_{r}, \quad \pi_{c}, \Phi\right) A=B$ or $\left(\pi_{r}, \pi_{c}\right) A=B$, respectively. Each of these isomorphism relations yields an equivalence relation decomposing the set of all sequences into disjoint isomorphism classes.

Theorem 2.3 Let $A$ and B be two $n \times m$ sequences of the same size. Then the isomorphism of $A$ and $B$ is decidable in $O\left(\min \left\{m n^{2}, m^{2} n\right\}\right)$ time.

The sets of all isomorphism of the same type and same formats form a group $G, S_{n} \times S_{m}, S_{n} \times S_{m} \times Z_{2}$ and $S_{n} \times S_{m} \times Z_{2} \times Z_{2}$ for permutation isomorphism, graph isomorphism and structure isomorphism, 
Tanka Nath Dhamala/On the Status of Irreducibility........

respectively. For each sequence $A$ and the group of any type above, the sets $\{\varphi \in G: \varphi(\mathrm{A})=\mathrm{A}\}$ and $\{\varphi(\mathrm{A}): \varphi \in \mathrm{G}\}$ are the stabilizer and orbit of A with the property $|\{\varphi \in G: \varphi(A)=A\}| \times|\{\varphi(A): \varphi \in G\}|$ $=|G|$.

The orbits are the isomorphism classes and the elements of the stabilizer of sequence $A$ are the automorphisms of $A$.

Therefore, given a system of distinct representatives SDR for each isomorphism classes, the total number of sequences is given by the algebraic class equation (Harborth 1999). However, the number of distinct automorphisms for $A$ is not known in general, an algebraic problem. The properties of sequence isomorphism play important roles for the enumeration of OSP (Braesel et al 1999a).

Theorem 2.4 Let $A$ and $B$ be two sequences in the same isomorphism class. Then sequence $A$ is irreducible if and only if the sequence $B$ is also irreducible.

\section{Irreducibility of Sequences}

We give different approaches of test and the current status of irreducibility in the OSP. Extensive studies consider the problem $O \| C_{\max }$ with respect to the irreducibility theory (Dhamala 2007, Andresen 2009).

The set of all irreducible sequences for the OSP $O 2 \| C_{\max }$ are presented in (Braesel \& Kleinau1996). Also, they obtain an optimal sequence in the set of all irreducible sequences. For every $A \in S^{I}{ }_{2 m}$ there exists a $k \in\{2, \ldots, m\}$ such that $\mathrm{A}$ can be obtained by a permutation of the columns of the following sequence

$\left(\begin{array}{ccccccc}1 & \ldots & k-1 & k & k+1 & \ldots & m \\ m-k+2 & \ldots & m & 1 & 2 & \ldots & m-k+1\end{array}\right)$

The main idea of their proof is to show that any sequence in this class is irreducible and any sequence not belonging to this class reduces to a sequence belonging to this class.

The total number of irreducible sequences for $O \mid n=$ $2 \mid C_{\max }$ is $m !(m-1)$ which is dominated by the total number of sequences. This justifies that only a small fraction of all sequences is irreducible.

\section{Comparability Graphs}

The decision problem whether a given sequence is reducible, similar or strongly reducible to another given sequence takes exponential time by using the definition. However, this can be solved by using the algorithms on the transitive closures for graphs which needs $O\left(n^{2} m^{2}\right)$ time to determine the transitive closure of a sequence graph and to check if $\left[G^{t r}{ }_{B}\right]$ is a subgraph of $\left[\mathrm{G}^{\mathrm{tr}}{ }_{\mathrm{A}}\right]$ (Braesel et al. 1999b).

Theorem 3.1 Let $A, B \in S_{n m}$. Then $A$ is similar, reducible or strongly reducible to $B$ for $O \| C_{\max }$ if and only if $\left[G^{t r}{ }_{B}\right]=\left[G^{t r}{ }_{A}\right],\left[G^{t r}{ }_{B}\right] \subseteq\left[G^{t r}{ }_{A}\right]$ or $\left[G^{t r}{ }_{B}\right]$ $\subset\left[G_{A}^{t r}\right]$, respectively.

Let $E_{d}$ be the set of all diagonal arcs in the transitive closure $G_{A}{ }_{A}=\left(S I J, E_{A}{ }^{t r}\right)$, and let $E_{A}=E_{A}{ }^{t r} \mid E_{d}$ be the set difference. Then the graph $\mathrm{G}_{\mathrm{A}}=\left(\mathrm{SIJ}, \mathrm{E}_{\mathrm{A}}\right)$ is such that $\left[\mathrm{G}_{\mathrm{A}}\right]=\mathrm{K}_{\mathrm{n}} \times \mathrm{K}_{\mathrm{m}}$ for any $\mathrm{n} \times \mathrm{m}$ sequence $A$. A sequence $A$ is irreducible if and only if there exists no comparability graph $G_{C}=\left(S I J, E_{C}\right)$ such that $\left[G_{A}\right] \subseteq G_{C} \subset\left[G^{t r}{ }_{A}\right]$. Assuming that a given graph $G$ contains no comparability graph $G_{C}$ with

$\left[G_{A}\right] \subseteq G_{C} \subset G$, they present an algorithm of complexity $O\left(n^{2} m^{2}\right)$ to test whether there is a sequence $A$ with $G=\left[G^{t r}{ }_{A}\right]$.

Several sufficient conditions for sequence reducibility which can be tested without computing the transitive closures of the associated sequence graphs are presented in (Braesel et al. 1999a, Harborth 1999). For example, an $n \times m$ sequence $\left[a_{i j}\right]$, where $\min \{n, m\} \geq 3$, having an operation $o_{i j}$ with $a_{i j} \geq n m-2$ is strongly reducible. Likewise, any sequence with $o_{i j}$ such that $o_{i j}$ has at least one successor but none of its successors in row $i$ or column $j$ has a direct predecessor outside row $i$ and column $j$, respectively, is strongly reducible to some sequence for $O \| C_{\max }$. Following result is applicable to investigate certain properties in OSP.

Theorem 3.2 Let $A$ be a sequence such that each job $i$ $\in\{1,2, \ldots, n\}$ is first processed on the same machine $j \in\{1,2, \ldots, m\}$. Then there exists a sequence $B \in S_{n m}$ such that $B \prec A$ for $C_{\text {maxx. }}$.

If one wish to test whether a given sequence can be strongly reduced to another sequence by deleting an operation and reinserting it as a sink or a source, it has to ensured that no new path is created in the latter 
sequence and at least one path is destroyed in the former one. This test can be performed in $O\left(n^{2} m^{2}\right)$ time and in $O\left(n^{2} m^{2}\right)$ space, given an $n \times m$ sequence (Braesel et al. 1999b). They prove that a sequence with only one sequence implication class is irreducible. In particular, any complete latin square is irreducible for the makespan objective. Therefore, if $n=m$, each rank minimal sequence is irreducible. But, in general, there exist rank minimal sequences which are reducible.

To check the reducibility of a sequence by the reversion of a certain set of arcs in it having more than a single implication class, one has to consider one implication class totally. In this reducibility test, for each subset of the set of sequence implication classes in given sequence, we have to construct a possibly reduced sequence from it by reversing the orientations of all arcs belonging to these sequence implication classes. This test depends on the number of sequence implication classes, which may cause exponential cost. In the worst case, if all operations belong to a single path, one has to check all $O\left(2^{n m 2}\right.$

${ }^{+m n 2}$ ) subsets of sequence implication classes (Braesel et al. 1999a). They illustrate that, the smaller ranks do not necessarily imply a less number of implication classes.

\section{Enumeration Procedures}

An enumeration algorithm which computes all irreducible sequences constructing inclusion minimal comparability graphs by successively inserting diagonal arcs into $\left[G_{A}\right]$ can be found in (Braesel et al. 1999a). Each sequence in such a set to minimize $C_{\max }$ is similar to exactly one sequence in this class, namely its reverse one. This algorithm constructs graphs $G$ such that $G=\left[G^{t r} A\right]$ for some sequence $A$. Number of diagonal arcs play crucial important role in this algorithm. For $\min \{n, m\} \geq 2$, a lower bound on the number of diagonal arcs of an $n \times m$ sequence on the complete operation set is of size $O\left(n^{2} m^{2}\right)$.

An insertion method for the enumeration of all sequences can be found in (Braesel \& Kleinau 1992). This method is modified and a new enumeration algorithm is presented in (Braesel et al. 1999a). In their algorithm, a set of nonisomorphic sequences is computed and, thereafter, tested for irreducibility. One sequence per isomorphic class is sufficient. They compute that the ratio between the number of irreducible sequences and all sequences decreases with growing $n$ and $m$.
A dominance relation is formulated as a mixed integer programming in (Tautenhahn \& Willenius 2000). They compute unavoidable sequences for small formats. Among seven classes of all $3 \times 3$ irreducible sequences only three of them are unavoidable in the sense that these together with their reverses are the unique optimal solutions for certain matrices of processing times. This set is the minimal one ensuring of at least one optimal solution. A sequence of biggest rank five among all $O 3 \mid n=$ $3 \mid C_{\max }$ irreducible sequences belongs to the class of unavoidable sequences. For the problem $O 3 \mid n=$ $2 \mid C_{\max }$, the minimal cardinality of a potentially optimal solution is 3 . There are two disjoint potentially optimal solutions of this cardinality. Thus, the minimal set is not unique.

The set of all latin squares does not guarantee the existence of an optimal solution for $O \| C_{\max }$. There exist irreducible sequences that are not rank minimal (Braesel et al. 1999a).

\section{Sequence Decomposition}

A generalized decomposition on irreducibility is introduced in (Dhamala 2002). For this, we consider an underlying $2 \times 2$ OS problem by the assignment of an operation to each part. In the $2 \times 2$ OSP, we hav2 irreducible (1 unavoidable) and 12 reducible sequences, namely, $\left(\begin{array}{ll}1 & 2 \\ 2 & 1\end{array}\right)$ and $\left(\begin{array}{ll}2 & 1 \\ 1 & 2\end{array}\right)$. Then, we partition an $n \times m$ sequence called the $A(i, j)$ decomposition $\mathrm{A}(\mathrm{i}, \mathrm{j})=\left(\begin{array}{cc}S_{i j} & S_{i, m-j} \\ S_{n-i, j} & S_{n-i, m-j}\end{array}\right)$.

In this partition, the $A(i, j)$-decomposition is a block decomposition where at least one part in $\left\{S_{i, j}, S_{i, m-j}\right.$, $\left.S_{n-i, j}, S_{n-i, m-j}\right\}$ is a block of at least $2 \times 2$ size. In the cases when any one of the sequences $S_{k, l}$ in $A(i, j)$ represents an $1 \times 1$ matrix, we denote it by $A(1,1)$ and we call an operation decomposition. In the $A(1$, 1) decomposition one of the blocks $S_{k, l}$ contains only one but arbitrary $\mathrm{o}_{\mathrm{uv}}$, without loss of generality, we assume that the block $S_{1,1}=\left(o_{11}\right)$.

Furthermore, consider the following three class representatives: $\left(\begin{array}{ll}1 & 2 \\ 2 & 2\end{array}\right),\left(\begin{array}{ll}1 & 2 \\ 2 & 3\end{array}\right)$ and $\left(\begin{array}{ll}1 & 2 \\ 4 & 3\end{array}\right)$, which are the lexicographically minimal in their structure isomorphic classes of $2 \times 2$ OSP. Then we 
Tanka Nath Dhamala/On the Status of Irreducibility........

construct the corresponding $A(\mathrm{i}, \mathrm{j})$ decompositions, denoted by type ${ }_{1}$, type 2 and type , $_{3}$, respectively.

An $n \times n$ irreducible sequence $A$ is said to satisfy the irr-decomposition property if it contains exactly $n$ sinks and there exists no other sequence $S \neq A_{-1}$ similar to the sequence $A$.

For the proof of Theorem 3.3, invariant property of irreducibility within each isomorphic class is applicable. Afterwards, it is shown that there is no any other nonsimilar sequence of the type 1 to which $A$ can be reduced. Neither it can be reduced to the sequence of any other types. It is conjectured that Theorem 3.3 holds even if the second condition of this property is violated. For this, the structure of similarity of sequences has to be understood better.

Theorem 3.3 Consider the $A(1,1)$ decomposition of type $_{1}$ in an $n \times n$ sequence $A$ where $S^{A}=S_{n-1, n-1}$ is irreducible for $\mathrm{O} \| \mathrm{C}_{\max }$. Then, $A$ is irreducible for $O \| C_{\max }$ if $S^{A}$ satisfies the irr-decomposition property.

As a consequence, any $A(1,1)$ decomposition of type $_{1}$ in an $(n+1) \times(n+1)$ sequence $A$, where $S_{n, n}$ is a latin square sequence of order $n$, is irreducible for $C_{\text {max }}$. Observe that $a_{i 1}$ and $a_{1 j}$ in this sequence are the permutations of $\{n+1, \ldots, 2 n\}$ for all $i, j \in\{2, \ldots$, $n+1\}$. Therefore, there exists an irreducible sequence of format $(n+1) \times(n+1)$ with maximal rank $2 n$ for $O \| C_{\text {max }}$.

Corollary 3.1 Any $A(\mathrm{i}, \mathrm{i})$-decomposition of type ${ }_{1}$, where all of its 4 partitions are latin squares of order at least 2 is an irreducible sequence for $C_{\max }$.

Any $A(1,1)$ decompositions of type ${ }_{2}$ and type tin $_{3}$ an $n \times m$ sequence $A$ is a strongly reducible sequence for $C_{\text {max }}$, see Theorem 3.2. However, a natural question is: do there exist irreducible sequences of type $e_{2}$ and type $_{3}$ in these block-decompositions? A positive answer is unlikely.

Note that the irr-decomposition property is not a necessary condition for the irreducibility of a sequence for $O \| C_{\max }$. If we consider an $A(i, j)$ decomposition for $i \geq 2$ and $j \geq 2$ with type ${ }_{1}$, then an example illustrates that there exists a reducible sequence even if all 4 partitions contain the same irreducible sequence. But, it is also possible to construct an irreducible sequence by considering the same irreducible sequence as its all 4 partitions. In the following, both sequences $A$ and $B$ are irreducible:
$A=\left(\begin{array}{llll}2 & 6 & 7 & 8 \\ 1 & 5 & 6 & 7 \\ 3 & 4 & 5 & 1 \\ 4 & 7 & 1 & 2\end{array}\right)$ and $B=\left(\begin{array}{llll}1 & 6 & 7 & 8 \\ 2 & 5 & 6 & 7 \\ 3 & 4 & 5 & 1 \\ 4 & 7 & 1 & 2\end{array}\right)$.
But the decompositions $\left(\begin{array}{ll}A & A \\ A & A\end{array}\right)$ and $\left(\begin{array}{cc}B & B \\ B & B\end{array}\right)$

are, respectively, irreducible and reducible for $C_{\max }$

\section{Generalized Numerical Data}

The concept of irreducibility for arbitrary release time $\mathrm{r}_{\mathrm{i}} \geq 0$, due date $d_{i} \geq 0$, weight $w_{i} \geq 0$ and $\mathrm{p}_{\mathrm{ij}} \geq$ 0 with respect to some regular objectives $\gamma$ can be found in (Willenius 2000).

A sequence $A \in \mathrm{S}_{\mathrm{nm}}$ is called $r$-reducible to $B \in \mathrm{S}_{n m}$, if $C_{\max }(B) \leq C_{\max }(A)$ for all instances of processing times $P$ and release dates $r=\left[r_{i}\right]$. A sequence $A \in$ $S_{n m}$ is called general reducible to $B \in S_{n m}$ if $C_{\mathrm{i}}(B) \leq$ $C_{\mathrm{i}}(A)$ for all jobs $i$ and all numerical data. Obviously, if $C_{i}(B) \leq C_{i}(A)$ for all jobs and all numerical data, then $\gamma(B) \leq \gamma(A)$ holds for all regular $\gamma$. The definitions of strong reducibility, similarity and irreducibility have been extended similarly.

A number of results in terms of comparability graphs and precedence relations between operations, and some interesting relations between the general reducibility and $r$-reducibility are established. For instance, for any two sequences $A$ and $B$, it holds that $B$ is r-reduced to $A$ if and only if $B_{-1}$ is g-reduced to $A_{-1}$.

\section{Conflict Resolution}

The theory of irreducibility for the OSP with respect to the H-comparability graph has been investigated and the complexity issues of the decision problem whether a given sequence is irreducible has been discussed in (Andresen 2009, Andresen \& Dhamala 2010). Two variants of algorithms, one with polynomial time complexity and the other an enumerative, have been proposed which differ depending on specific characteristics of the diagonal edges of the corresponding H-comparability graph of the given sequence. For computational results we refer to, [LiSA, 2010]. 
Nepal Journal of Science and Technology 11 (2010) 205 - 214

These results relay on the role of the diagonal edges which have to be removed as a set rather than as a particular member. Main idea behind this is to destroy a path, which has to be broken between two extended sequence implication classes. One of the main issues while doing this process is that the diagonal edges, which belong to the irreducible sequences, should not be removed and the problem of merging two implication classes occurs when an edge play a role of conflict.

The theory of reducibility has been considered since last 20 years from both theoretical as well as practical view points. The set of all irreducible sequences is potentially (universally) optimal for the OSP though it is not the minimal one. The existence of unique minimal solution is unlikely in general. The nonsimilar irreducible sequences yield a solution set of still smaller cardinality, but this space is not good understood yet. Determination of this set is extremely important. One of the strong motivations of studying the theory of irreducibility comes from the fact that only a very small fraction among all sequences is irreducible.

No polynomial time algorithm is known for the decision whether a sequence is irreducible though a number of polynomial tests have been presented. The good properties of this set has to be detected which helps to develop exact and heuristic algorithms in OS. Development of new constructive and iterative neighborhood structures in this smaller set would be a constructive step.

By defining a number of graph structures in the space of the induced Hamming comparability graph, different reduction strategies which can be tested in polynomial time have been presented in recent outcomes. A number of open problems have been explored from this research. In coming issues we would like to discuss on various conjectures whose validity decide whether the irreducibility problem belongs to the polynomial solvable class or to the $N P$-class, respectively.

\section{References}

Andresen, M. and T.N. Dhamala. 2010. New algorithms on the reducibility of open shop sequences minimizing the Makespan. Submitted to Mathematical Methods of Operations Research.
Andresen, M. 2009. On the complexity of reducibility problems through $\mathrm{H}$ comparability graphs, Ph.D. thesis. University of Magdeburg, Germany.

Andresen, M., H. Braesel, M. Plauschin, and F. Werner 2008. Using simulated annealing for open shop scheduling with sum criteria. Global optimization: Focus on simulated annealing, I-Techonline.

Braesel, H., M. Harborth, and P. Willenius. 2001. Isomorphism for digraphs and sequences of shop scheduling problems. Journal of combinatorial mathematics and combinatorial 37:115-128.

computing

Braesel, H., M. Harborth, T. Tautenhahn, and P. Willenius. 1999a. On the set of solutions of the open shop problem. Annals of operations research 92:241-263.

Braesel, H., M. Harborth, T. Tautenhahn and P. Willenius. 1999b. On the hardness of the classical job shop problem. Annals of operations 92:265-279.

Braesel, H. and M. Kleinau. 1996. New Steps in the Amazing world of sequences and schedules. Mathematical methods of operations research 43:195-214.

Brasel, H. and M. Kleinau. 1992a. On number problems for the open shop problems. System modeling and optimization, proceedings of the 15th IFIP conference, Zurich, Switzerland, Springer Verlag, pp. $145-155$.

Braesel, H. and M. Kleinau. 1992b. On the number of feasible solutions of the open shop problem - An application of special latin rectangles. optimization 23:251-260.

Braesel, H. 1990. Latin rectangle in scheduling theory. Dissertation B, University of Magdeburg, Germany.

Dhamala, T.N. 2010. Reducibility problems of open shop sequences minimizing the makespan. Proceedings of the 19th workshop on discrete optimization, Holzhau, Germany. pp. 57-60.

Dhamala, T.N. and M.H. Lee. 2008. Structures of sequences in the classical open shop schedul-ing problem. Proceedings of the 10th international workshop on multimedia signal processing and transactions, Chonbuk National University. pp. 15-24.

Dhamala, T. N. 2007. On the Potentially Optimal Solutions of Classical Shop Scheduling Problems. International Journal of Operations Research 4(2):80-89. 
Tanka Nath Dhamala/On the Status of Irreducibility........

Dhamala, T.N. 2002. Shop scheduling solutionspaces with algebraic characterizations. $\mathrm{PhD}$ thesis, University of Magdeburg, Germany, Shaker Verlag.

Gonzalez T. and S. Sahni. 1976. Open shop scheduling to minimize finish times. Journal of the association of computing machinery 23:665-679.

Harborth, M. 1999. Structural investigation of shop scheduling problems: Number problems, potential optimality and new enumeration algorithms. Ph.D. thesis. University of Magdeburg, Germany.

Kleinau, M. 1993. On the structure of shop scheduling problems: Number problems, reducibility and complexity. Ph.D. thesis. University of Magdeburg, Germany.
Prins, C. 1994. An overview of scheduling problems arising in satellite communications. Journal of operations research society 40: 611-623.

Sotskova, N. 2001. Optimal scheduling with uncertainty in the numerical data on the basis of a stability analysis. Ph.D. thesis. University of Magdeburg, Germany.

Tautenhahn, T. and P. Willenius. 2000. How many sequences solve an open shop problem preprint, University of Magdeburg, Germany.

Tautenhahn, T. 2000. Irreducible sequences on tree-like operation sets. Preprint, University of Magdeburg, Germany.

Willenius, P. 2000. Irreducibility theory in scheduling theory. Ph.D. Thesis. University of Magdeburg, Germany, Shaker Verlag.

http://lisa.math.uni-magdeburg.de: LiSA - A Library of Scheduling Algorithm 
Nepal Journal of Science and Technology 11 (2010) 205 - 214 